\title{
Nonparametric estimation of effect heterogeneity in rare events meta-analysis: bivariate, discrete mixture model
}

\author{
Dankmar Böhning and Susan Martin \\ Mathematical Sciences and \\ Southampton Statistical Sciences Research Institute \\ University of Southampton, UK \\ email:d.a.bohning@soton.ac.uk \\ Patarawan Sangnawakij \\ Department of Mathematics and Statistics \\ Faculty of Science and Technology \\ Thammasat University, Thailand \\ Katrin Jensen, Walailuck Böhning and Heinz Holling \\ Statistics and Quantitative Methods \\ Faculty of Psychology and Sports Science \\ University of Münster, Germany
}

August 1, 2020

\section{Contents}

1 Introduction 2

2 Case study 2

3 The log-linear model with heterogeneity 3

4 Diagnosing heterogeneity 5

5 Case study (continued) 5

6 Discussion and conclusions 6 


\begin{abstract}
a short abstract ....

Key words: Heterogeneity Variance; Jensen's inequality; Nonparametric Mixture Models; Meta-analysis; Rare events.
\end{abstract}

\title{
1 Introduction
}

The paper considers the following situation in meta-analysis. In $k$ independent studies, counts of events are observed in an intervention and control group. This setting can be described by a count random variable $Y_{i j}$. The index $i$ indicates the study $i$ for $i=1,2, \ldots, k$, where $k$ denotes the number of available studies. Also, $j=1$ denotes an intervention group and $j=0$ a control group. $Y_{i j}$ stand for the number of events in study $i$ and group $j$, whereas $T_{i j}$ stands for the person-time at risk in study $i$ and group $j$. The latter is considered as non-random and reduces to the number at risk $n_{i j}$, if all members in study $i$ share the same person-time. Furthermore, conditional upon study $i$ we have that $E\left(Y_{i j}\right)=\lambda_{i j} T_{i j}$, where $\lambda_{i j}$ denotes the event occurrence risk in study $i$ and group $j$. We are interested often in settings where the probability of no events is large, so that often low frequency counts such as 0,1 , or 2 are observed. In this connection, the Poisson assumption comes into play which assumes that

$$
Y_{i j} \sim \operatorname{Po}\left(\lambda_{i j} T_{i j}\right)
$$

for study $i$ and group $j$, where $\operatorname{Po}(\theta)$ denotes the Poisson distribution with the density $e^{-\theta} \theta^{y} / y$ ! for count $y=0,1,2, \ldots$ Under assumption (1), the mean and variance of $Y_{i j}$ are $E\left(Y_{i j}\right)=\lambda_{i j} T_{i j}=\operatorname{Var}\left(Y_{i j}\right)$.

We emphasize that (1) is conditional upon the study $i$ and treatment group $j$ and uses as such a study-group specific parameter $\lambda_{i j}$. Hence it is a reasonable but untestable assumption as we only have one count $Y_{i j}$ observed per study and group combination. In addition, the count $Y_{i j}$ will be often small to the extrem of having no events in one or both groups.

\section{Case study}

We will now illustrate the various estimators at the example of a case study. Cooper et al. (2003) mentioned a systematic review of the effectiveness of prophylactic antibiotic treatment on infectious complications in women undergoing caesarean delivery, originally published by Smaill and Hofmeyr (2002). The data included 61 studies with counts of occurrence of wound infection as outcome in women undergoing caesarean delivery. The intervention group used prophylactic antibiotics whereas the control group was placebo or no prophylactic antibiotics. The data showed sample sizes with an average of 80 persons per trial in the treatment arm and 63 persons per trial in the control. The occurrence of wound infection was observed relatively rarely. Many of the component 
studies were small in size and there were zero events in each of two arms, especially in the treatment group. If a traditional inverse variance-weighted average method is used, the double-zero or single-zero study would have to be excluded before the analysis because the risk ratio $\widehat{R R}_{i}=\frac{Y_{i 1} / T_{i 1}}{Y_{i 0} / T_{i 0}}$ is undefined.

Using the data on prophylactic antibiotics in caesarean treatment, the results of a meta-analysis on the risk ratio is presented using a forest plot in Figure 1 (one study is excluded due to the above mentioned issue of occurrence of zero counts). Overall, a women undergoing caesarean delivery appears to have a lower risk for infectious complications if in the prophylactic antibiotic treatment group relative to being in placebo or in the no prophylactic antibiotic treatment group. One of the central questions in a meta-analysis of effects is whether there is homogeneity of effect. In the next section we will present a modelling approach that can help answering this question.

\section{The log-linear model with heterogeneity}

Given the Poisson model (1) we may reparameterize the mean as follows:

$$
\log E\left(Y_{i j}\right)=\log \lambda_{i j}+\log T_{i j}=\alpha_{i}+\beta_{i} \times j+\log T_{i j} .
$$

The reparameterisation has the benefit that the log-risk ratio in the $i$-th study is given by $\beta_{i}$ and corresponds to $\log R R_{i}$ ). In addition, heterogeneity can be now separated due to baseline heterogeneity - the variability in the intercept $\alpha_{i}$ - and the heterogeneity in the effect measure - the variability in the slope $\beta_{i}$. The situation of effect homogeneity is characterized by $\beta_{i}=0$ for all studies $i=1, \cdots, k$. To model heterogeneity the typical generalized liner mixed model approach takes $\alpha_{i} \sim N\left(\alpha, \sigma_{\alpha}^{2}\right)$ and $\beta_{i} \sim N\left(\beta, \sigma_{\beta}^{2}\right)$. Instead assuming a normal (or other parametric) distribution we leave the distribution of $\left(\alpha_{i}, \beta_{i}\right)$ unspecified. It belongs to the classics of nonparametric maximum likelihood estimation that the maximum likelihood estimator maximizing the mixture log-likelihood with mixing distribution $Q$

$$
\ell(Q)=\sum_{i, j} \log \int p\left(y_{i j} ; \exp \left(\alpha_{i}+\beta_{i} \times j+\log T_{i j}\right)\right) Q\left(d \alpha_{i}, d \beta_{i}\right)
$$

is always discrete Lindsay $(1982,1995)$. Here $p(y ; \lambda)=\exp (-\lambda) \lambda^{y} / y$ ! is the Poisson discrete mass function for $y=0, \cdots$ and $\lambda>0$. Hence there is no limitation of generality if we replace (3) by

$$
\ell(Q)=\sum_{i, j} \log \sum_{s=1}^{S} p\left(y_{i j} ; \exp \left(\alpha_{s}+\beta_{s} \times j+\log T_{i j}\right)\right) q_{s} .
$$

The log-likelihood (4) is evidently a discrete mixture log-likelihood with weights $q_{1}, \cdots, q_{S}$ being positive and summing up to 1 . Unfortunately, it is not known which values for $S$ should be chosen. This is known as the number of components 


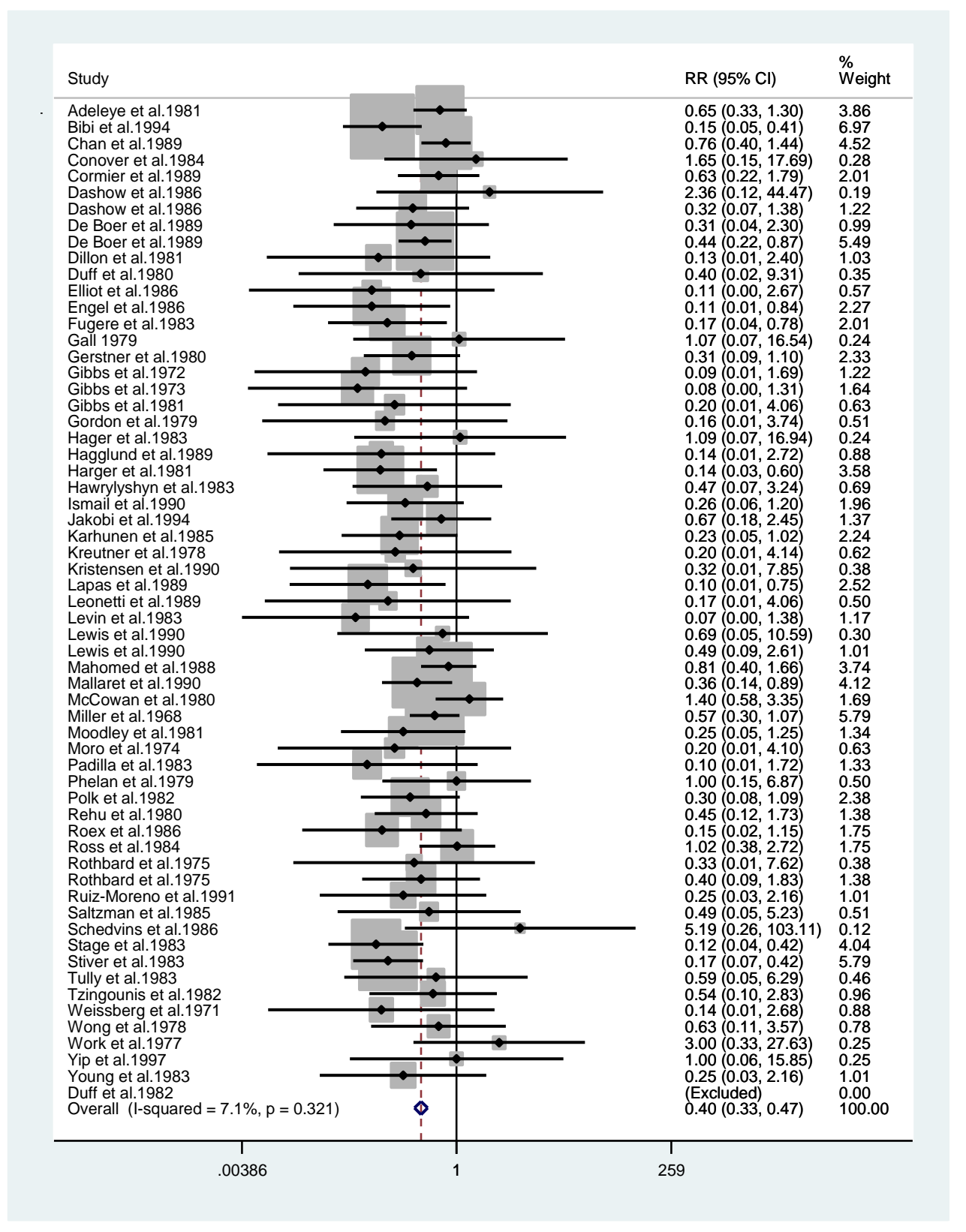

Figure 1: Forest plot of prophylactic antibiotics in caesarean section 
problem. A typical solution is to start with $S=1$ and then sequentially increase the number of components by one until not further increase in the log-likelihood is found. For a given value of $S$, the log-likelihood (4) is maximized using the EM algorithm (Dempster, Laird and Rubin 1977; McLachlan and Krishnan 2007).

We will denote the maximum likelihood estimate of the parameters $\alpha_{s}, \beta_{s}$ and $q_{s}$ for $s=1, \cdots, S$ as

$$
\hat{Q}=\left(\begin{array}{ccc}
\hat{\alpha}_{1} & \cdots & \hat{\alpha}_{S} \\
\hat{\beta}_{1} & \cdots & \hat{\beta}_{S} \\
\hat{q}_{1} & \cdots & \hat{q}_{S}
\end{array}\right) .
$$

Note that $\hat{Q}$ is a mixing distribution jointly on the intercept $\alpha$ and the slope (log-risk ratio) $\beta$. Having the maximum likelihood estimate available we are then able to give a nonparametric estimate of the heterogeneity variance of the log-relative risk as

$$
\hat{\tau}^{2}=\sum_{s=1}^{S}\left(\hat{\beta}_{s}-\bar{\beta}\right)^{2} \hat{q}_{s},
$$

where $\bar{\beta}=\sum_{s=1}^{S} q_{s} \hat{\beta}_{s}$. This variance is of particular interest in meta-analysis as its size indicates the amount of heterogeneity in effect size across studies. Of course, other variances such as the baseline heterogeneity variance in the $\alpha_{s}$ can be considered.

\section{Diagnosing heterogeneity}

\section{Case study (continued)}

We continue the discussion of the case study. Our interest is structure and form of the heterogeneity in the log-risk ratio across studies. In Table 1 we consider in its top part the modeling of the mixture distribution of the bivariate intercept and slope parameters $\left(\alpha_{s}, \beta_{s}\right)$ where in the bottom part we consider only heterogeneity in the baseline parameter $\alpha_{s}$ while keeping the slope parameter homogeneous. We can see from Table 1 that the best model for the bivariate mixture is given by $S=2$ (according to the BIC) or $S=3$ (according to the AIC) components, respectively. For the univariate mixture with homogeneity in the log-risk ratio the best model is given with $S=2$ components (both, AIC and BIC, select identically). It is interesting that according to the BIC the best model is the homogeneous risk ratio model with two component heterogeneity in the baseline parameter. According to the AIC the best model is a risk ratio heterogeneity model with 3 components. We interpret this that we have here a meta-analysis with a mild form of effect heterogeneity. 
Table 1: Model evaluation under heterogeneity and homogeneity of effect

\begin{tabular}{cccccc}
$S$ & log-likelihood & AIC & BIC & $\hat{\tau}^{2}$ & $\bar{\beta}$ \\
\hline \multicolumn{7}{c}{ bivariate mixture } \\
1 & -359.2 & 722.5 & 726.8 & 0 & -0.96 \\
2 & -289.9 & 589.7 & 600.5 & 0.04 & -0.77 \\
3 & -284.3 & 584.6 & 601.8 & 0.99 & -1.02 \\
4 & -283.4 & 588.8 & 612.6 & 1.03 & -1.02 \\
\multicolumn{7}{c}{ univariate mixture keeping $\beta_{s}=\beta$} \\
1 & -359.2 & 722.5 & 726.8 & - & -0.96 \\
2 & -291.7 & 591.3 & 599.9 & - & -0.64 \\
3 & -289.9 & 591.8 & 604.8 & - & -0.66 \\
4 & -289.9 & 595.8 & 613.1 & - & -0.66
\end{tabular}

\section{Discussion and conclusions}

\section{References}

[Böhning and Sangnawakij(2020)] Böhning, D. And SAngnawakiJ, P. (2020). The identity of two meta-analytic likelihoods and the ignorability of double-zero studies. Biostatistics 0, 1-7. DOI: 10.1093/biostatistics/kxaa004.

[Cochran(1954)] Cochran, W. (1954). Some methods for strengthening the common $\chi^{2}$ tests. Biometrics 10, 417-451.

[Cooper and others(2003)] Cooper, N. J., Abrams, K. R., Sutton, A. J., Turner, D. and Lambert, P. C. (2006). Use of bayesian methods for Markov modelling in cost-effectiveness analysis: an application to taxane use in advanced breast cancer. Journal of the Royal Statistical Society. Series A (Statistics in Society) 166, 389-405.

[Cui and others(2017)] Cui, Y., Li, C., Xu, Z., Wang, Y., Sun, Y., Xu, H., LI, Z. AND SUn, Y. (2017). Robot-assisted versus conventional laparoscopic operation in anus-preserving rectal cancer: a meta-analysis. Therapeutics and clinical risk management 13, 1247-1257.

[Dragomir(1992)] Dragomir, S. S. (1992). Some refinements of Jensen's inequality. Journal of Mathematical Analysis and Applications 168, 518-522.

[Efthimiou and others(2019)] Efthimiou, O., RüCker, E., Schwarzer, G., Higgins, J. P. T., Egger, M. And Salanti, G. (2019). Network metaanalysis of rare events using the Mantel-Haenszel method. Statistics in Medicine 38, 2992-3012.

[Facchinetti and others(2020)] Facchinetti, G., D'Angelo, D., Piredda, M., Petitti, T., Matarese, M., Oliveti, A. and Marinis, M. G. 
(2020). Continuity of care interventions for preventing hospital readmission of older people with chronic diseases: a meta-analysis. International journal of nursing studies 101, 1-10.

[Fieller(1932)] Fieller, E. C. (1932). The distribution of the index in a bivariate normal distribution. Biometrika 24, 428-440.

[Gottlieb and others(2019)] Gottlieb, M., Holladay, D. And PeKsa, G. D. (2019). Ultrasound-assisted lumbar punctures: a systematic review and meta-analysis. Academic Emergency Medicine 26, 85-96.

[Greenland and Robins(1985)] Greenland, S. and Robins, J. M. (1985). Estimation of common effect parameter from sparse follow up data. Biometrics 41, 55-68.

[Jewell(2004)] JEWELl, N. P. (2004). Statistics for Epidemiology, CRC Press: Boca Raton.

[Huang and others(2017)] HuAng, L., Yin, Y., Yang, L., Wang, C., Li, Y. AND ZHOU, Z. (2017). Comparison of antibiotic therapy and appendectomy for acute uncomplicated appendicitis in children: a meta-analysis. JAMA Pediatrics 171, 426-434.

[Kirk and others(2019)] Kirk, A. H. P., YAng, J., Sim, W. C., ChiA, L. Y. X. AND LAU, Y. (2019). Systematic review of the effect of topical application of human breast milk on early umbilical cord separation. Journal of Obstetric, Gynecologic, and Neonatal Nursing 48, 121-130.

[Klingenberg(2013)] KLIngEnBERG, K. (2013). A new and improved confidence interval for the Mantel-Haenszel risk difference. Statistics in Medicine 33, 2968-2983.

[Landis and others(2005)] LAndis, J. R., Sharp, T. J., Kuritz, S. J. And Koch, G. G. (2005). Mantel-Haenszel Methods, Encyclopedia of Biostatistics. John Wiley \& Sons: Chichester.

[Lui(2004)] Lui, K. J. (2004). Statistical Estimation of Epidemiological Risk, John Wiley \& Sons: Chichester.

[Mantel and Haenszel(1959)] Mantel, N. And Haenszel, W. (1959). Statistical aspects of the analysis of data from retrospective studies of disease. Journal of the National Cancer Institute 22, 719-748.

[R Core Team(2020)] R CoRE TEAM. (2020). R: a language and environment for statistical computing. $R$ Foundation for Statistical Computing, Vienna: Austria. http://www.R-project.org/

[Robins and others(1986)] Robins, J., Greenland, S. And Breslow, N. E. (1986). A general estimator for the variance of the Mantel-Haenszel odds ratio. American Journal of Epidemiology 124, 719-723. 
[Rossel and Taffé(2019)] Rossel, R. P. And Taffé, P. (2019). Meta-analysis of rare events under the assumption of a homogeneous treatment effect. Biometrical Journal 61, 1557-1574.

[Rothman and others(2008)] Rothman, K. J., Greenland, S., and Lash, T. L. (2008). Modern Epidemiology, Lippincott Williams \& Wilkins: Philadelphia.

[Rücker and others(2020)] Rücker, G., Nikolakopoulou, A., Papakonstantinou, T., Salanti, G., Riley, R. D. and Schwarzer, G. (2020). The statistical importance of a study for a network meta-analysis estimate. Research Square 0, 1-18. DOI: 10.21203/rs.2.15672/v2.

[Sato(1989)] Sato, T. (1989). On the variance estimator for the MantelHaenszel risk difference. Biometrics 45, 1323-1324.

[Schulze and others(2003)] Schulze, R., Holling, H. And Böhning, D. (2003). Meta-analysis: New Developments and Applications in Medical and Social Sciences, Hogrefe \& Huber: Göttingen.

[Smaill and Hofmeyr(2002)] Smaill, F. And Hofmeyr, G. J. (2002). Antibiotic prophylaxis for cesarean section. Cochrane Database of Systematic Reviews 3, 1-71.

[Webster and Hewett(2019)] Webster, K. E. And Hewett, T. E. (2019). What is the evidence for and validity of return-to-sport testing after anterior cruciate ligament reconstruction surgery? a systematic review and meta-analysis. Sports Medicine 49, 917-929. 